\title{
Ab initio engineering of Fano resonances
}

\author{
Benjamin Gallinet and Olivier J. F. Martin \\ Nanophotonics and Metrology Laboratory, Swiss Federal Institute of Technology Lausanne \\ (EPFL), 1005 Lausanne, Switzerland
}

\begin{abstract}
In this work, we pave the route towards the engineering of strong and spectrally sharp Fano resonances in plasmonic nanostructures and derive analytical formulas for their line shape as a function of their electromagnetic response. Contrary to the original work of Fano, the formalism proposed here includes losses in the materials composing the system. As a result, a more general formula is obtained for the response of the system and general conclusions for the determination of the resonance parameters are drawn, in particular on its width and asymmetry. Using a surface integral simulation technique for electromagnetic scattering on three-dimensional individual and periodic nanostructures, we numerically validate our model for structures that are currently under extensive investigation in the plasmonic and metamaterial communities. The insights into the physical comprehension of Fano resonances gained this way will be of great interest for the design of plasmonic sensing platforms and metamaterials.
\end{abstract}

Keywords: Plasmons, Fano Resonances, Electromagnetically-Induced Transparency, Surface Integrals, Method of Moments

\section{INTRODUCTION}

Asymmetric resonances display unique features, compared to their symmetric Lorentzian counterpart, and are currently the subject of considerable research efforts in photonic and plasmonic nanostructures. Fano resonances exhibit a very strong sensitivity to changes of the local environment as well as a sharp spectral dispersion. ${ }^{1,2}$ In plasmonics, they arise when a non-radiative (dark) mode interacts with a radiative (bright) mode. The control of their line shape for each specific application remains a challenging task, and requires a theory to understand the underlying physical mechanisms. Numerical and experimental data are usually fitted and analyzed with the two coupled mechanical oscillators ${ }^{3,4}$ or other intuitive phenomenological models. ${ }^{5-9}$ As result, it is for example not well understood in a realistic plasmonic system composed of several interacting particles how the individual modes and their coupling affect the overall Fano-like resonance of the system.

In this work, we pave the route towards the engineering of strong and spectrally sharp Fano resonances in plasmonic nanostructures and metamaterials. From an ab initio theory for asymmetric resonances ${ }^{10}$ we derive analytical expressions for the resonance parameters and discuss how they are influenced by the electromagnetic interactions between the individual modes. In Section 2, we recall the main results of the electromagnetic theory of Fano resonances. In Section 3, we derive closed-form expressions for the resonance line shape parameters and in Section 4 we use these predictions to engineer the optical response of dolmen nanostructures supporting Fano resonances, which are currently under extensive investigation in the plasmonic and metamaterial communities.

\section{ELECTROMAGNETIC THEORY OF FANO RESONANCES}

In this section, a general theory for Fano resonances in photonic and plasmonic nanostructures is developed. We consider the electromagnetic scattering on a dielectric or metallic object in a dielectric background. The scatterer supports a non-radiative (dark) mode interacting with a continuum of radiative (bright) waves. A harmonic time-dependence for the fields $\mathbf{E}=\mathbf{E}_{0} e^{-i \omega t}$ is assumed throughout. The relative dielectric permittivity $\epsilon(\mathbf{r}, \omega)$

Further author information: (Send correspondence to O.J.F.M.)

O.J.F.M.: E-mail: olivier.martin@epfl.ch

B.G.: E-mail: benjamin.gallinet@epfl.ch

Plasmonics: Metallic Nanostructures and Their Optical Properties IX, edited by Mark I. Stockman Proc. of SPIE Vol. 8096, 80961R - (C) 2011 SPIE · CCC code: 0277-786X/11/\$18 · doi: 10.1117/12.893631

Proc. of SPIE Vol. 809680961 R-1 
is generally complex and has frequency dispersion. The frequency dependent electric field $\mathbf{E}$ satisfies the wave equation

$$
\epsilon^{-1}(\mathbf{r}, \omega) \nabla \times \nabla \times \mathbf{E}(\mathbf{r}, \omega)-\frac{\omega^{2}}{c^{2}} \mathbf{E}(\mathbf{r}, \omega)=0 .
$$

To simplify notation, in the following the dependence of the electric field $\mathbf{E}$ on $\omega$ is kept implicit. Let us assume that the scatterer permittivity is given by a Drude model with plasma frequency $\omega_{p}$; the scaling law of Maxwell's equations allows then to scale all frequency units by $\omega_{p}$ and length units by $2 \pi c / \omega_{p}$. We introduce the generally complex and frequency dependent differential operator $\underline{\underline{\mathcal{M}}}_{\omega}$ defined by

$$
\underline{\underline{\mathcal{M}}}_{\omega} \mathbf{E}(\mathbf{r})=\frac{c^{2}}{\epsilon(\mathbf{r}, \omega)} \nabla \times \nabla \times \mathbf{E}(\mathbf{r})
$$

The wave equation can be written for a vectorial wave function $|\mathbf{E}\rangle$

$$
\left(\underline{\underline{\mathcal{M}}}_{\omega}-\omega^{2} \underline{\underline{I}}\right)|\mathbf{E}\rangle=0
$$

where $\underline{I}$ is the identity operator. The inner product is defined by

$$
\left\langle\mathbf{E}_{1} \mid \mathbf{E}_{2}\right\rangle=\int \mathbf{E}_{1}^{*}(\mathbf{r}) \cdot \mathbf{E}_{2}(\mathbf{r}) d^{3} \mathbf{r} .
$$

Following Feshbach, Bhatia and Temkin, we introduce the orthogonal projection operators $P$ and $Q$ splitting the wave function into a radiative (bright) and a non-radiative (dark) part. ${ }^{11,12}$ Any wave function $|\mathbf{E}\rangle$ can be decomposed as $|\mathbf{E}\rangle=Q|\mathbf{E}\rangle+P|\mathbf{E}\rangle$ where only $P|\mathbf{E}\rangle$ satisfies the radiation condition. Equation (3) becomes

$$
\left(\underline{\underline{\mathcal{M}}}_{\omega}-\omega^{2} \underline{\underline{I}}\right)(Q|\mathbf{E}\rangle+P|\mathbf{E}\rangle)=0 .
$$

We consider a unique non-radiative mode $\left|\mathbf{E}_{d}\right\rangle$, defined to be eigenfunction of the projector to non-radiative modes $Q\left|\mathbf{E}_{d}\right\rangle=\left|\mathbf{E}_{d}\right\rangle$, and to satisfy $Q \underline{\underline{\mathcal{M}}}_{\omega_{d}} Q\left|\mathbf{E}_{d}\right\rangle=z_{d}^{2}\left|\mathbf{E}_{d}\right\rangle$ and $\left|\left\langle\mathbf{E}_{d} \mid \mathbf{E}_{d}\right\rangle\right|^{2}=1$. Taking material losses into account, the quantity $z_{d}=\omega_{d}+i \gamma_{d}$ is generally complex. Its real part $\omega_{d}$ is the mode resonance frequency, and $\gamma_{d}$ its intrinsic damping. The system is studied in the vicinity of the resonance frequency $\omega_{d}$. In this frequency range, we assume that the space of eigenfunctions of $Q$ is spanned by $\left|\mathbf{E}_{d}\right\rangle$ only, ${ }^{13}$ so that $Q=\left|\mathbf{E}_{d}\right\rangle\left\langle\mathbf{E}_{d}\right|$. The radiative (bright) wavefunction $\left|P \mathbf{E}_{b}\right\rangle$ is defined to satisfy the following homogeneous wave equation in the radiative region:

$$
\left(P \underline{\underline{\mathcal{M}}}_{\omega} P-\omega^{2}\right)\left|P \mathbf{E}_{b}\right\rangle=0 .
$$

The Green's function $\underline{\underline{G}}_{b}$ of Eq. (6) satisfies:

$$
\left(P \underline{\underline{\mathcal{M}}}_{\omega} P-\omega^{2}\right) \underline{\underline{G}}_{b}=\underline{\underline{I}} .
$$

Most plasmonic nanostructures and metamaterials are embedded in a dielectric medium, allowing us to assume that the permittivity is real and non dispersive in the radiative region, and therefore that $P \underline{\underline{\mathcal{M}}} \omega$ is self-adjoint. The solutions of Eq. (6) form a continuum on which the Green's function $\underline{\underline{G}}_{b}$ of Eq. (6) can be expanded:

$$
\underline{\underline{G}}_{b}=\frac{1}{\pi} \mathcal{P} \int d \omega^{\prime} \frac{\left|P \mathbf{E}_{b}\left(\omega^{\prime}\right)\right\rangle\left\langle P \mathbf{E}_{b}\left(\omega^{\prime}\right)\right|}{\omega^{\prime 2}-\omega^{2}},
$$

where $\mathcal{P}$ denotes the Cauchy principal value of the integral. The expression of the Green's function in Eq. (8) depends on the normalization of the continuum $\left|P \mathbf{E}_{b}\right\rangle$. It can be shown that the total field, as a solution of Eq. (3), is a combination of the dark mode with the continuum of radiative waves. It satisfies: ${ }^{10}$

$$
|\tilde{\mathbf{E}}\rangle=\left|P \mathbf{E}_{b}\right\rangle+\frac{\left\langle\mathbf{E}_{d}|\underline{\underline{\mathcal{M}}} \omega| P \mathbf{E}_{b}\right\rangle}{z_{d}^{2}-\omega^{2}+\omega_{d} \Delta}\left(\underline{\underline{G}}_{b} P \underline{\underline{\mathcal{M}}}_{\omega}\left|\mathbf{E}_{d}\right\rangle-\left|\mathbf{E}_{d}\right\rangle\right),
$$

where

$$
\Delta=-\left\langle\mathbf{E}_{d}\left|\underline{\underline{\mathcal{M}}}_{\omega} P \underline{\underline{G}}_{b} P \underline{\underline{\mathcal{M}}}_{\omega}\right| \mathbf{E}_{d}\right\rangle / \omega_{d}
$$


The wavefunction $|\tilde{\mathbf{E}}\rangle$ is related to $|\mathbf{E}\rangle$ but does not have the same asymptotic behavior. The wavefunction $|\mathbf{E}\rangle$ must have the same norm as $P\left|\mathbf{E}_{b}\right\rangle$ in the far-field (i.e. $\left.\left.|P| \mathbf{E}\right\rangle\left.\right|^{2}=|P| \mathbf{E}_{b}\right\rangle\left.\right|^{2}$ ). With the explicit expression of the Green's function [Eq. (8)] and using the residue theorem, one obtains From Eq. (9):

$$
|P \tilde{\mathbf{E}}\rangle=\left|P \mathbf{E}_{b}\right\rangle\left[1-\frac{\left|\left\langle\mathbf{E}_{d}\left|\underline{\underline{\mathcal{M}}}_{\omega}\right| P \mathbf{E}_{b}\right\rangle\right|^{2}}{2 \omega\left(z_{d}^{2}-\omega^{2}+\omega_{d} \Delta\right)} i\right]
$$

Let us define the intrinsic damping parameter:

$$
\Gamma_{i}=\frac{\left|\left\langle\mathbf{E}_{d}|\underline{\underline{\underline{\mathcal{M}}}} \omega| P \mathbf{E}_{b}\right\rangle\right|^{2} \gamma_{d} \omega_{d}}{\omega\left(\omega_{d}^{2}-\omega^{2}+\omega_{d} \Delta\right)^{2}}
$$

the resonance width:

$$
\Gamma=\frac{\left|\left\langle\mathbf{E}_{d}|\underline{\underline{\mathcal{M}}}| P \mathbf{E}_{b}\right\rangle\right|^{2}}{2 \omega\left(1-\Gamma_{i}\right)}
$$

and the reduced frequency:

$$
\kappa=\left(\omega^{2}-\omega_{d}^{2}-\omega_{d} \Delta\right) / \Gamma .
$$

In order for $|\mathbf{E}\rangle$ to have the same normalization in the far-field as $\left|\mathbf{E}_{b}\right\rangle$, it is related to $|\tilde{\mathbf{E}}\rangle$ by

$$
|\mathbf{E}\rangle=\cos \eta|\tilde{\mathbf{E}}\rangle /\left(1-\Gamma_{i}\right),
$$

where $\operatorname{cotan} \eta=\kappa$, considering $\gamma_{d} \ll \omega_{d}$ and neglecting any second order contribution. We have $P|\mathbf{E}\rangle=$ $P\left|\mathbf{E}_{b}\right\rangle \exp (i \eta)$, meaning that the phase of $|\mathbf{E}\rangle$ shifts rapidly by $\sim \pi$ in a frequency region $\Gamma$ around the resonance position $\omega_{d}^{2}+\omega_{d} \Delta$. The parameters $\omega_{d}$ and $\Delta$ determine its spectral position. Its width $\Gamma$ is influenced by the field overlap between the continuum $\left|P \mathbf{E}_{b}\right\rangle$ and the non-radiative mode $\left|\mathbf{E}_{d}\right\rangle$, which is a direct effect of their interference. We consider now a transition operator $T$ from an initial state $|g\rangle$ to a final state $|\tilde{\mathbf{E}}\rangle$, which can be interpreted as the response of an optical system to an external excitation, such as the Local Density of States (LDOS), ${ }^{14}$ the forward scattering cross-section ${ }^{15}$ or the reflectance of a two-dimensional array. From Eq. (9):

$$
\frac{\langle g|T| \tilde{\mathbf{E}}\rangle}{\left\langle g|T| P \mathbf{E}_{b}\right\rangle}=1-\frac{\left|\left\langle\mathbf{E}_{d}\left|\underline{\underline{\mathcal{M}}}_{\omega}\right| P \mathbf{E}_{b}\right\rangle\right|^{2}}{2 \omega\left(z_{d}^{2}-\omega^{2}+\omega_{d} \Delta\right)\left(1-\Gamma_{i}\right)} q,
$$

with

$$
q=2 \omega\left(1-\Gamma_{i}\right) \frac{\left\langle g|T| \mathbf{E}_{d}\right\rangle-\left\langle g\left|T \underline{\underline{G}}_{b} P \underline{\underline{\mathcal{M}}}_{\omega}\right| \mathbf{E}_{d}\right\rangle}{\left(\left\langle\mathbf{E}_{d}\left|\underline{\underline{\mathcal{M}}}_{\omega}\right| P \mathbf{E}_{b}\right\rangle\right) *\left\langle g|T| P \mathbf{E}_{b}\right\rangle} .
$$

The parameter $q$ is given by the ratio between the optical response of the perturbed non-radiative mode and the continuum. Assuming $q$ is real, one gets from Eqs. (15) and (16) the ratio of the optical response of the total field $|\mathbf{E}\rangle$ to the one of the continuum $\left|P \mathbf{E}_{b}\right\rangle$ :

$$
\sigma=\frac{|\langle g|T| \mathbf{E}\rangle|^{2}}{\left|\left\langle g|T| P \mathbf{E}_{b}\right\rangle\right|^{2}}=a \frac{(\kappa+q)^{2}+b}{\kappa^{2}+1}
$$

where $b=\kappa^{2} \Gamma_{i}^{2} q^{2}\left(1-\Gamma_{i}\right)^{-2}$ and $a=\left(1-\Gamma_{i}\right)^{-2}$. Equation (18) introduces a family of line shapes as shown in Fig. 1, with an additional parameter $b$ describing the resonance contrast. ${ }^{10}$

\section{DARK AND BRIGHT MODES INTERFERENCE}

The continuum of radiative waves $\left|P \mathbf{E}_{b}\right\rangle$ is usually generated from a bright plasmon mode. In this particular case, each resonance parameter in Eq. (18) has a closed-form expression which will be derived in this section. These expressions reveal how the different resonance parameters are influenced by the individual modes and their coupling. 

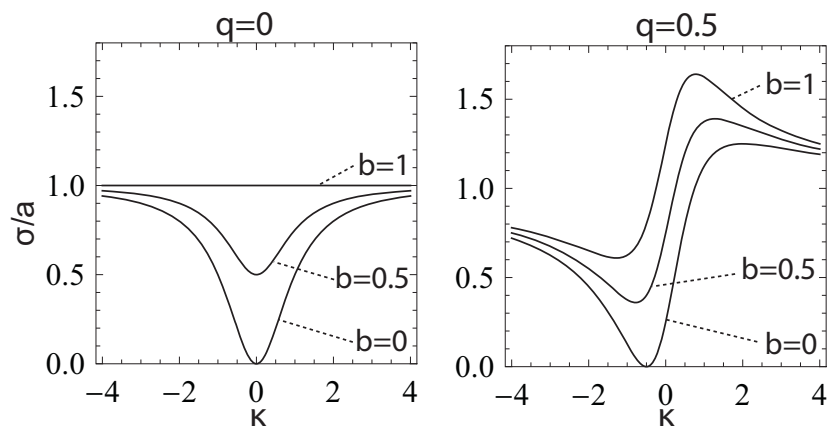

Figure 1. Resonance shape function $\sigma / a$ as a function of the reduced frequency $\kappa=\left(\omega^{2}-\omega_{d}^{2}-\omega_{d} \Delta\right) / \Gamma$ for different values of the asymmetry parameter $q$ and the screening parameter $b$ [Eq. (18)].

It is assumed that all the parameters are independent of the frequency in a spectral region around the resonance, and can be evaluated at $\omega_{d}$. From Eqs. (8) and (10), the Fano resonance frequency shift from $\omega_{d}$ is given by the following integral:

$$
\begin{aligned}
\Delta & =-\frac{1}{\pi \omega_{d}} \mathcal{P} \int d \omega^{\prime} \frac{\left|\left\langle P \mathbf{E}_{b}\left(\omega^{\prime}\right)|\underline{\underline{\mathcal{M}}}| \mathbf{E}_{d}\right\rangle\right|^{2}}{\omega^{\prime 2}-\omega_{d}^{2}} \\
& =-\frac{\left|\left\langle P \mathbf{E}_{b}\left(\omega_{s}\right)|\underline{\underline{\mathcal{M}}}| \mathbf{E}_{d}\right\rangle\right|^{2} \Gamma_{s}^{2}}{\pi \omega_{d}} \mathcal{P} \int d \omega^{\prime} \frac{1}{\left(\omega^{\prime 2}-\omega_{d}^{2}\right)\left[\left(\omega^{\prime 2}-\omega_{s}^{2}\right)^{2}+\Gamma_{s}^{2}\right]},
\end{aligned}
$$

where $\omega_{s}$ is the bright mode's resonance frequency and $\Gamma_{s}$ its resonance width. In order to calculate this integral, we assume that $\omega^{\prime}$ is a complex variable. The integration path in the complex plane is a closed path following the real axis and a half circle of infinite radius on the lower complex plane, including poles at $\sqrt{\omega_{s}^{2}+i \Gamma_{s}}$ and $\omega_{d}$. Using the residue theorem, one obtains:

$$
\Delta=\frac{c^{2}\left(\omega_{d}^{2}-\omega_{s}^{2}\right) \Gamma_{s}}{2 \omega_{d}^{2}\left[\left(\omega_{d}^{2}-\omega_{s}^{2}\right)^{2}+\Gamma_{s}^{2}\right]}
$$

where $c=\left|\left\langle P \mathbf{E}_{b}\left(\omega_{s}\right)|\mathcal{M}| \mathbf{E}_{d}\right\rangle\right|$ is the coupling strength between the dark and bright modes. The sign of $\Delta$ is determined by the frequency difference between the two modes, and its magnitude linearly depends on the coupling strength, which is a signature of weak coupling. From Eqs. (12) and (21), the intrinsic damping parameter $\Gamma_{i}$ becomes:

$$
\Gamma_{i}=\frac{4 \gamma_{d}\left[\left(\omega_{d}^{2}-\omega_{s}^{2}\right)^{2}+\Gamma_{s}^{2}\right] \omega_{d}^{2}}{c^{2}\left(\omega_{d}^{2}-\omega_{s}^{2}\right)^{2}}
$$

The magnitude of $\Gamma_{i}$ is driven by the ratio $\gamma_{d} / c^{2}$, meaning that the effect of intrinsic losses is critical if the coupling between the dark and bright modes is not sufficient. From Eq. (13), the resonance width becomes:

$$
\Gamma=\frac{c^{2} \Gamma_{s}^{2}}{2 \omega_{d}\left[\left(\omega_{d}^{2}-\omega_{s}^{2}\right)^{2}+\Gamma_{s}^{2}\right]\left(1-\Gamma_{i}\right)}
$$

It is determined by the coupling strength between the modes and also affected by the intrinsic damping parameter $\Gamma_{i}$. The asymmetry parameter $q$ depends on a transition element $T$, which we consider from now one to describe the reflectance of an array of nanostructures. In these conditions, the dark mode is characterized by $\left\langle g|T| \mathbf{E}_{d}\right\rangle=0$. A similar integral as in Eq. (19) is calculated to obtain an analytical expression for the asymmetry parameter:

$$
q=\left(1-\Gamma_{i}\right) \frac{\omega_{d}^{2}-\omega_{s}^{2}}{\Gamma_{s}},
$$

which is also a function of the frequency detuning between the two modes. For other transition elements, the resulting expression for the $q$ parameter would be different (Section 2). In addition, the parameter $\Gamma_{i}$ is 


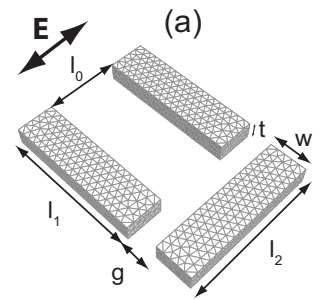

(c)

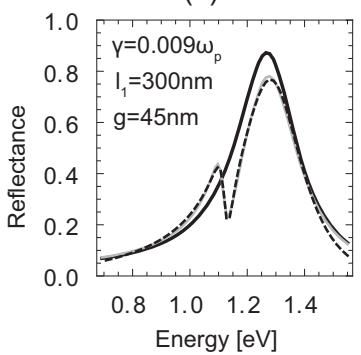

(b)

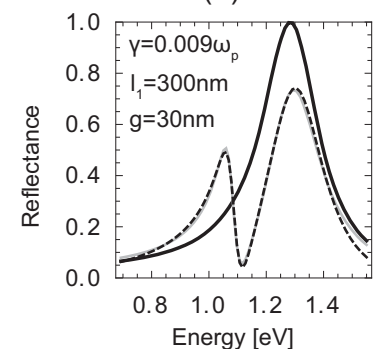

(d)

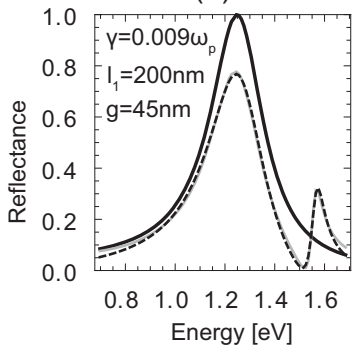

(e)

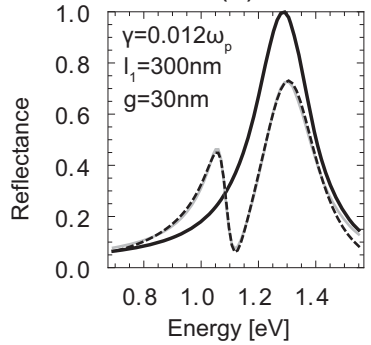

Figure 2. Reflectance of dolmen-type plasmonic nanostructures at normal illumination. The dolmens are arranged in a two-dimensional array of period $500 \mathrm{~nm}$ and placed in vacuum. The material is chosen to satisfy Drude model with plasma frequency $\omega_{p}=1.37 \times 10^{16} \mathrm{~s}^{-1}$ and damping $\gamma$. (a) Discretization and geometry of the unit cell with dimensions $w=40 \mathrm{~nm}, l_{0}=160 \mathrm{~nm}, t=80 \mathrm{~nm}$ and $l_{2}=300 \mathrm{~nm}$. (b) to (e): black dashed-numerical simulations; gray-fit with Eq. (26); black-background resonance with parameters $A, \omega_{s}$ and $\Gamma_{s}$ extracted from the fit. The following values of the fitting parameters are obtained: (b) $A=1.00, \omega_{s}=1.28 \mathrm{eV}, \Gamma_{s}=0.31 \mathrm{eV}^{2}, \omega_{a}=1.08 \mathrm{eV}, \Gamma_{a}=0.07 \mathrm{eV}^{2}, q=-0.94$, and $\mathrm{b}=0.30 ;$ (c) $A=0.93, \omega_{s}=1.27 \mathrm{eV}, \Gamma_{s}=0.33 \mathrm{eV}^{2}, \omega_{a}=1.12 \mathrm{eV}, \Gamma_{a}=0.05 \mathrm{eV}^{2}, q=-0.37$, and $\mathrm{b}=0.66 ;(\mathrm{d})$ $A=1.00, \omega_{s}=1.25 \mathrm{eV}, \Gamma_{s}=0.33 \mathrm{eV}^{2}, \omega_{a}=1.55 \mathrm{eV}, \Gamma_{a}=0.08 \mathrm{eV}^{2}, q=1.30$, and b=0.15; (e) $A=1.00, \omega_{s}=1.29 \mathrm{eV}$, $\Gamma_{s}=0.31 \mathrm{eV}^{2}, \omega_{a}=1.08 \mathrm{eV}, \Gamma_{a}=0.08 \mathrm{eV}^{2}, q=-0.87$, and $\mathrm{b}=0.40$.

responsible for damping in case of high losses or low coupling. Other components of intrinsic losses are described by the screening parameter $b$ :

$$
b=4 \frac{\gamma_{d}^{2} q^{2}}{\Delta^{2}}=\frac{16 \gamma_{d}^{2}\left(1-\Gamma_{i}\right)^{2} \omega_{d}^{4}\left[\left(\omega_{d}^{2}-\omega_{s}^{2}\right)^{2}+\Gamma_{s}^{2}\right]^{2}}{\Gamma_{s}^{4} c^{4}} .
$$

The screening parameter is mainly influenced by the ratio $\gamma_{d}^{2} / c^{4}$. If the coupling between the two modes is too weak compared to intrinsic losses, the parameter $b$ increases, which in turn screens the Fano resonance (Fig. 1).

\section{OPTICAL RESPONSE OF DOLMEN NANOSTRUCTURES}

As seen in Section 3, the resonance parameters are determined by the modes resonance frequency and damping, as well as by their coupling. In this section, we illustrate these effects by numerical calculations, and show how the line shape of Fano-like resonances can be controlled in plasmonic nanostructures. We consider an array of dolmen-type plasmonic nanostructure made of three metallic beams arranged as in Fig. 2(a). The two parallel beams support a quadrupolar dark mode, while the third perpendicular beam supports a dipolar bright mode. The interference between the two modes leads to a Fano-like resonance. ${ }^{16-20}$ In Fig. 2(b) to (e), the reflectance spectra of an array of dolmen nanostructures are calculated with a surface integral formulation method for periodic nanostructures. ${ }^{21,22}$ In order to compute reflectance spectra, the scattered electric field is sampled sufficiently far away from the array. The spectra are fitted with the following analytical expression, describing the modulation of the bright mode's reflectance by the asymmetric line shape of Eq. (18):

$$
R(\omega)=\frac{A^{2} \Gamma_{s}^{2}}{\left(\omega^{2}-\omega_{s}^{2}\right)^{2}+\Gamma_{s}^{2}} \frac{\left(\omega^{2}-\omega_{a}^{2}+q \Gamma\right)^{2}+b \Gamma^{2}}{\left(\omega^{2}-\omega_{a}^{2}\right)^{2}+\Gamma^{2}}
$$


The fit is performed by variation of the 7 parameters $A, \omega_{s}, \Gamma_{s}, \omega_{a}, \Gamma_{a}, q$ and $b$. In order to fulfill energy conservation, the amplitude $A$ of the bright mode's reflectance is constrained to values inferior to 1 . The parameters $A, \omega_{s}$ and $\Gamma_{s}$ are used in Fig. 2(b) to (e) to reconstruct the symmetric resonance of the bright mode. The total reflectance clearly appears as an asymmetric modulation of the bright mode's resonance. We will now discuss how this modulation can be engineered with geometrical and physical parameters in the structure.

The central frequency $\omega_{a}$ is determined by the dark mode resonance frequency $\omega_{d}$ and the coupling shift $\Delta$ [Eq. (21)]. In Fig. 2(d), the pair of beams has been made shorter as compared to Fig. 2(b) in order to increase $\omega_{d}$. The asymmetric modulation is then blue-shifted to the other shoulder of the bright mode's resonance. The coupling between the dark and bright modes is tuned with the gap distance between the beams pair and the individual beam. In Fig. 2(c), the two modes are moved further apart, which decouples the two modes and in turn shifts the modulation frequency $\omega_{a}$ to higher values. On the other hand, the bright mode's frequency has the opposite behavior, which is a signature of weak coupling. In the far-field, the asymmetry parameter is a function of the frequency detuning between the two modes [Eq. (24)]. In Fig. 2(d), the dark mode's resonance frequency $\omega_{d}$ is shifted to the high frequency shoulder of the bright mode's resonance. As a result, the asymmetry parameter flips its sign from negative to positive values, compared to Fig. 2(c).

The resonance width $\Gamma$ is mainly determined by the modes coupling strength $c$ [Eq. (23)], which can be seen in Fig. 2(c) where decoupling the modes renders the modulation sharper. The quantity $c$ also appears in a ratio $\gamma_{d} / c^{2}$ with the intrinsic losses constant (as the imaginary part of the dark mode's resonance frequency) in the asymmetry and screening parameters. As an effect, if the intrinsic losses are too large compared to the coupling, the resonance is screened and cannot be observed. Quantitatively, the screening parameter $b$ increases and the asymmetry parameter $q$ vanishes from Fig. 2(b) to Fig. 2(c) where the two modes are decoupled. Another effect of the ratio $\gamma_{d} / g^{2}$ is observed when increasing the constant $\gamma_{d}$ [Fig. 2(e)]: The modulation depth and asymmetry parameter decrease, while the width of the resonance increases, as predicted by the analytical expressions in Section 3. In order to obtain a strong and sharp Fano resonances, both the screening parameters and the spectral width have to be engineered. This can be done by controlling the modes coupling and the intrinsic losses in the structure.

\section{CONCLUSION}

We have derived from Maxwell's equations an analytical expression for Fano resonances in plasmonic nanostructures and metamaterials using Feshbach formalism. This theory relies on the interference between a radiative (bright) mode and non-radiative (dark) mode established in the entire system. An asymmetric line shape is

derived for the response of the system, characterized by four parameters which are controlled by the dark and bright mode's resonance frequencies and coupling strength, as well as intrinsic losses. These parameters are the resonance frequency, the spectral width, the asymmetry parameter and the screening parameter. The behavior of these parameters has been verified by fitting the analytical formula to numerical simulations in dolmen nanostructures. This work enables the control of the line shape of asymmetric resonances in plasmonic systems based on Maxwell's equations. The insights into the physical comprehension of Fano resonances gained this way will be of great interest for the design of plasmonic sensing platforms and metamaterials.

\section{ACKNOWLEDGMENTS}

Funding from CSEM and CCMX-Fanosense as well as stimulating discussion with M. Schnieper and A. Stuck are gratefully acknowledged.

\section{REFERENCES}

1. Miroshnichenko, A. E., Flach, S., and Kivshar, Y. S., "Fano resonances in nanoscale structures," Rev. Mod. Phys. 82, 2257-2298 (2010).

2. Luk'yanchuk, B., Zheludev, N. I., Maier, S. A., Halas, N. J., Nordlander, P., Giessen, H., and Chong, C. T., "The Fano resonance in plasmonic nanostructures and metamaterials," Nat. Mater. 9, 707-715 (2010).

3. Alzar, C. L. G., Martinez, M. A. G., and Nussenzveig, P., "Classical analog of electromagnetically induced transparency," Am. J. Phys. 70, 37-41 (2002). 
4. Joe, Y. S., Satanin, A. M., and Kim, C. S., "Classical analogy of fano resonances," Phys. Scr. 74, 259-266 (2006).

5. Fan, S. and Joannopoulos, J. D., "Analysis of guided resonances in photonic crystal slabs," Phys. Rev. B 65, $235112(2002)$.

6. Fan, S. H., Suh, W., and Joannopoulos, J. D., "Temporal coupled-mode theory for the Fano resonance in optical resonators," J. Opt. Soc. Am. A-Opt. Image Sci. Vis. 20, 569-572 (2003).

7. Christ, A., Ekinci, Y., Solak, H. H., Gippius, N. A., Tikhodeev, S. G., and Martin, O. J. F., "Controlling the Fano interference in a plasmonic lattice," Phys. Rev. B 76, 201405 (2007).

8. Ruan, Z. and Fan, S., "Temporal Coupled Mode Theory for Fano Resonance in Light Scattering by a Single Obstacle," J. Phys. Chem. C 114, 7324-7329 (2010).

9. Verellen, N., Van Dorpe, P., Huang, C., Lodewijks, K., Vandenbosch, G. A. E., Lagae, L., and Moshchalkov, V. V., "Plasmon Line Shaping Using Nanocrosses for High Sensitivity Localized Surface Plasmon Resonance Sensing," Nano Lett. 11, 391-397 (2011).

10. Gallinet, B. and Martin, O. J. F., "Ab initio theory of Fano resonances in plasmonic nanostructures and metamaterials," Physical Review B 83, 235427 (2011).

11. Feshbach, H., "A unified theory of nuclear reactions .2.," Ann. Phys. 19, 287-313 (1962).

12. Bhatia, A. K. and Temkin, A., "Line-shape parameters for P-1 Feshbach resonances in He and Li+," Phys. Rev. A 29, 1895-1900 (1984).

13. O’Malley, T. F. and Geltman, S., "Compound-atom states for 2-electron systems," Phys. Rev. 137, A1344 (1965).

14. Novotny, L. and Hecht, B., [Principles of Nano-Optics], Cambridge University Press (2006).

15. Bohren, C. F. and Huffman, D. R., [Absorption and Scattering of Light by Small Particles], WileyInterscience (1983).

16. Zhang, S., Genov, D. A., Wang, Y., Liu, M., and Zhang, X., "Plasmon-induced transparency in metamaterials," Phys. Rev. Lett. 101, 047401 (2008).

17. Verellen, N., Sonnefraud, Y., Sobhani, H., Hao, F., Moshchalkov, V. V., Van Dorpe, P., Nordlander, P., and Maier, S. A., "Fano resonances in individual coherent plasmonic nanocavities," Nano Lett. 9, 1663-1667 (2009).

18. Liu, N., Langguth, L., Weiss, T., Kaestel, J., Fleischhauer, M., Pfau, T., and Giessen, H., "Plasmonic analogue of electromagnetically induced transparency at the drude damping limit," Nat. Mater. 8, 758-762 (2009).

19. Liu, N., Weiss, T., Mesch, M., Langguth, L., Eigenthaler, U., Hirscher, M., Soennichsen, C., and Giessen, H., "Planar metamaterial analogue of electromagnetically induced transparency for plasmonic sensing," Nano Lett. 10, 1103-1107 (2010).

20. Pryce, I. M., Aydin, K., Kelaita, Y. A., Briggs, R. M., and Atwater, H. A., "Highly Strained Compliant Optical Metamaterials with Large Frequency Tunability," Nano Lett. 10, 4222-4227 (2010).

21. Gallinet, B. and Martin, O. J. F., "Scattering on plasmonic nanostructures arrays modeled with a surface integral formulation," Photonic Nanostruct. 8, 278-284 (2010).

22. Gallinet, B., Kern, A. M., and Martin, O. J. F., "Accurate and versatile modeling of electromagnetic scattering on periodic nanostructures with a surface integral approach," J. Opt. Soc. Am. A 27, 2261-2271 (2010). 